\title{
Stalker: una teoria psicodinamica
}

Roberto Infrasca ${ }^{1}$

${ }^{1}$ Dipartimento di Psichiatria, ASL5 La Spezia

\begin{abstract}
Stalking is a common social problem, that can cause psychological and social damage in its victims. It can be defined as a pattern of repeated, unwanted attention, harassment, and contact. This behaviour is often driven by psychiatric disorder, therefore it is important that mental health professional can have the instruments to assess and manage it properly. Starting from the data observed during the personal experience of the Author, this article outlines a structured profile of the stalker's personality and its complexity. Psychopathological characteristics, psychosocial history and mental dynamics of the stalker are described. The aim is to provide information about factors contributing to stalking, as well as diagnostic issues that should be considered in the assessment and treatment of these patients.
\end{abstract}

Keywords: stalking, persecution, psychosocial dynamics, mental health professionals

Stalking: a psychodinamic theory

Pratica Medica \& Aspetti Legali 2010; 4(3): 103-112

\section{INTRODUZIONE}

Lo stalking rappresenta un insieme di comportamenti attraverso i quali un soggetto impone a un altro ripetute intrusioni (pedinare, sorvegliare, sostare nelle vicinanze, tentare approcci con la vittima), unitamente a comunicazioni assillanti (invio di lettere, di e-mail, telefonate e messaggi) $[1,2]$. Alcuni ricercatori definiscono questo comportamento come un ostinato, malevolo, ripetitivo e opprimente inseguimento attuato nei confronti di una persona, che entra in una situazione di paura poiché sente minacciata la sua sicurezza personale [3-5].

Galeazzi e Curci hanno introdotto il concetto di sindrome delle molestie assillanti per definire "un insieme di comportamenti ripetuti e intrusivi di sorveglianza e controllo, di ricerca di contatto e di comunicazione nei confronti di una vittima che risulta infastidita e/o allarmata da tali attenzioni e comportamenti», condizioni che rimandano alla presenza di una patologia comunicativa e relazionale nell'attore di tale atteggiamento [6].

Analizzando la letteratura internazionale, e basandosi sullo studio di molti casi clinici e giudiziari, altri Autori hanno disegnato le caratteristiche generalmente riscontrate nel molestatore assillante (stalker), individuando i tratti fondamentali nel rifiuto, nell'ossessione, nella fantasia, nel narcisismo e nella manipolazione interpersonale [7].

A livello epidemiologico, alcune ricerche mettono in luce che l' $80 \%$ delle vittime di stalking è costituito da donne e il $20 \%$ da uomini; il $13 \%$ ha un'età inferiore ai 18 anni, il 45\% è compreso tra 18-29 anni, il 24\% tra 30-39, e 18\% ha oltre 40 anni [8]. L'Osservatorio Nazionale Stalking (ONS - Milano) in collaborazione con il CoISP (sindacato di Polizia) ha analizzato un campione di 9.600 persone appartenenti a 16 regioni (periodo 2001/2007). Dall'analisi dei dati emerge che circa il $20 \%$ della popolazione ha vissuto o sta vivendo questa infausta esperienza, l' $80 \%$ è di sesso femminile, il $70 \%$ ha avuto esiti psico-relazionali frequentemen- 


\begin{tabular}{|lc|}
\multicolumn{1}{|c|}{ Attività } & Frequenza (\%) \\
\hline Telefonate indesiderate & 56 \\
\hline Approcci intrusivi diretti & 56 \\
\hline Pedinamenti & 49 \\
\hline Sostare nelle vicinanze della vittima & 35 \\
\hline Sorveglianza continua & 31 \\
\hline E-mail, fax e lettere indesiderate & 19 \\
\hline
\end{tabular}

Tabella I. Rituali predefiniti dello stalking identificati dalla ricerca dell'Osservatorio Nazionale Stalking [9]

te gravi, il $17 \%$ delle vittime sporge denuncia, nel $90 \%$ dei casi esiste una rapporto di conoscenza tra stalker e vittima, l'incidenza maggiore del fenomeno stalking sembra situarsi nel centro nord.

Inoltre, questa ricerca mette in risalto che tale comportamento si avvale di rituali (pre)definiti quali quelli elencati in Tabella I.

Infine, le dinamiche dello stalking sono attuate nel 55\% circa nelle relazione di coppia, nel 25\% nei condomini, nel $15 \%$ sul posto di lavoro e nel $5 \%$ in famiglia tra figli, fratelli e genitori [9].

Un altro capitolo importante del fenomeno stalking emerge nella professione delle donne (sesso maggiormente colpito) vittime del comportamento argomentato, vale a dire quelle definite professioni di aiuto (educatori, infermieri, assistenti sociali, medici, psicologi, ecc.). Di fatto, una ricerca evidenzia che le donne che lavorano in queste professioni sono più soggette a stalking $(78,6 \%$ contro il $21,4 \%$ di uomini) [10]. I ricercatori hanno individuato due possibili motivazioni dell'alta incidenza dello stalking in questo ambito professionale:

- queste figure professionali entrano in contatto con i bisogni profondi di aiuto delle persone e possono più facilmente diventare oggetto di meccanismi proiettivi, legami psichici provenienti da arcaiche relazioni interiorizzate;

- lo stalking può essere una richiesta di attenzione o una ricerca vendicativa di un risarcimento affettivo.

Muovendo dal presupposto che la personalità stalking evidenzia un'organizzazione multidimensionale cui concorrono una serie di fattori, il presente lavoro vuole proporre un profilo psicodinamico di tale fenomeno, volto a dare una risposta - per quanto possibile chiara ed esaustiva - alla sua complessità. Tale modalità si avvale dell'esperienza clinica fatta dall'Autore con alcuni soggetti che rientrano nel fenomeno stalking. Infine, in considerazione del fatto che la maggioranza degli stalker sono di sesso maschile [11], le argomentazioni svolte nel presente studio si riferiscono solo a tale sesso.

\section{CARATTERISTICHE PERSONOLOGICHE E PSICOPATOLOGICHE DELLO STALKER}

La personalità stalker si evidenzia come una struttura fragile e immatura, caratterizzata dall'interazione di alcuni tratti psico-comportamentali specifici, sistematicamente ritrovati. La miscelazione dinamica di tali fattori e la loro concomitanza tenderebbero così a comporre il quadro deviante $\mathrm{e} i$ particolari comportamenti messi in atto. Di seguito presentiamo queste organizzazioni, unitamente alla loro connessione con lo stalking.

\section{ATTACCAMENTO}

Il principale significato dell'attaccamento è rappresentato dalla sopravvivenza: l'individuo, in situazioni di precarietà o di pericolo (fisica e/o psichica), viene messo nella condizione di essere accudito e rassicurato. Bowlby sostiene che, qualora il sistema di attaccamento si attivi ma non possa ottenere soddisfazione - vale a dire la vicinanza e l'aiuto da parte della figura significativa - tale processo tenderà a disattivarsi automaticamente [12].

Il meccanismo di disattivazione dell'attaccamento viene osservato specialmente nella protratta separazione del bambino dalla figura d'attaccamento. In tale situazione nel bambino si osserva la sequenza di tre distinte risposte psico-comportamentali. Una prima fase - definita di protesta nella quale il bambino tenta di ribellarsi alla separazione attraverso la rabbia, il pianto e la ricerca della persona perduta, viene sostituita da uno stato di disperazione e di tristezza nel quale il bambino sembra avere perso la speranza del ritorno della figura, e infine una terza fase nella quale si assiste al distacco, evento che segnala l'avvenuta separazione emozionale da tale figura.

Lo stile di attaccamento contiene al suo interno una serie di sentimenti e strategie specifiche per affrontare e regolare il disagio nelle situazioni esterne (Modelli Operativi Interni), e quello che in tali circostanze l'individuo deve aspettarsi da sé e dagli altri (vale a dire la capacità di regolare le emozioni e le aspettative nei confronti della risposta), l'immagine di sé, dell'altro e della relazione tra i due, unitamente alle emozioni che si formano in questo ambito [13].

La tipologia dell'attaccamento si evidenzia come una delle più importanti variabili implicate nel fenomeno argomentato. In letteratura l'attaccamento di tipo insicuro-evitante o disorganizzato viene proposto come l'organizzazione di interazione relazionale che aumenta notevolmente la propensione al comportamento stalking [14,15]. Alcuni 
ricercatori suggeriscono che il gruppo stalking dimostra un insufficiente modello di attaccamento interpersonale, evidenzia limitate capacità di formare e mantenere relazioni, è emotivamente labile e instabile [16].

Altri studi sostengono che l'attaccamento disorganizzato è associato a comportamenti successivi altamente aggressivi [17], ad alcuni disturbi psichiatrici [18], alla violenza coniugale [19], unitamente al fatto che la correlazione più significativa tra attaccamento e psicopatologia si ha con il Disturbo di Personalità Borderline [20].

La personalità dello stalker, quindi, potrebbe essere caratterizzata da un blocco alla fase di protesta, stadio infantile caratterizzato da rabbia intensa e ribellione per la mancanza (fisica ed emotivo-affettiva) della figura materna [12], impedimento che produrrebbe l'incapacità dell'individuo di raggiungere la fase depressiva (stadio del distacco emozionale), unica modalità per elaborare il vissuto di "perdita".

\section{LUTTO}

Situata in uno spazio prossimale o distale dalla nascita, l'esperienza della perdita dell'oggetto si evidenzia quale elemento che caratterizza e innesca il processo psicodinamico definito "lutto". Saranno le diverse vicissitudini e percorsi seguiti da tale processo a determinare la (graduale) risoluzione dello stesso, oppure l'aggancio psicopatologico del soggetto all'interno di interminabili e compulsivi circuiti di sofferenza psichica.

Principalmente, il concetto di perdita dell'oggetto non riguarda solo le situazioni nelle quali "qualcosa" di obiettivo non esiste più, bensì tutte le situazioni nelle quali viene sperimentata la perdita di un fattore ritenuto soggettivamente significativo, poiché investito di una forte connotazione emotivo-affettiva.

L'esperienza di perdita include così - senza differenze significative - la persona (lutto tradizionale), un'idea o un ideale, un'aspirazione, un sogno, un oggetto fisico, una sicurezza affettiva, un'aspettativa, una garante situazione, la fiducia (di base nel bambino), un bisogno illusorio, ecc. L'ampiezza e la dimensione psicodinamica della "perdita" spaziano quindi in ambiti estremamente più profondi e complessi di quelli tradizionalmente e culturalmente definiti.

Precoci esperienze di perdita "dell'oggetto d'amore", generalmente situate nel periodo dell'infanzia o prossimali, non possono essere elaborate date le condizioni strutturali di fragilità e immaturità dell'lo [21]. Il bambino sviluppa così un rapporto con l'oggetto cattivo in cui prevalgono odio, rancore, ostilità, distruttività, grave insufficienza relazionale. Questa reazione di aggressività distruttiva e di profonda rabbia non è visibile nel bambi-

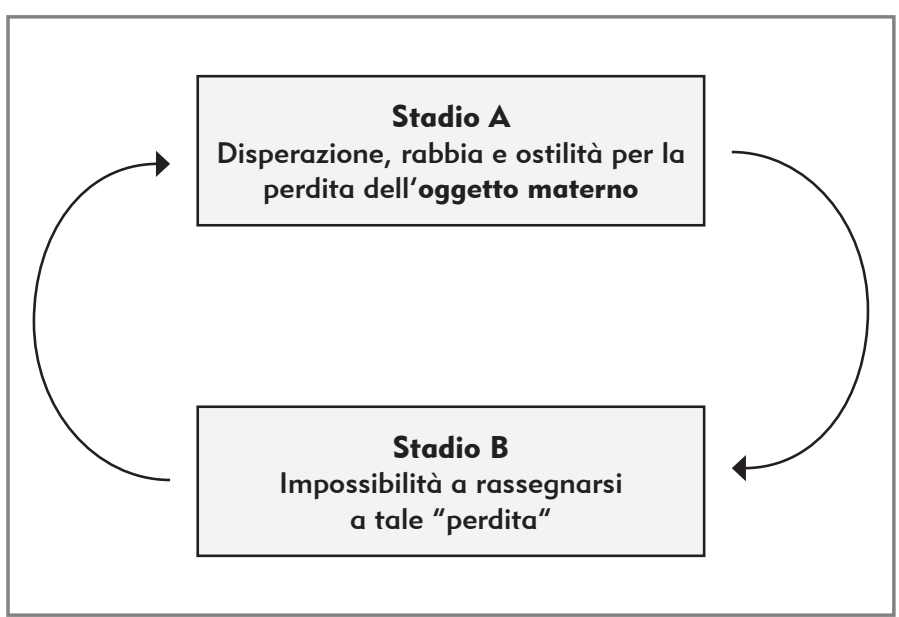

Figura 1. Rappresentazione dell'andamento "circolare" del lutto nello stalker

no perché la fragilità dell'lo non gli permette di evidenziare questi tratti, potenziali connotazioni originarie che la crescita renderà invece sempre più evidenti.

Nella personalità stalker è quindi possibile ipotizzare che l'elaborazione del lutto infantile conseguente alla perdita dell'oggetto madre (attaccamento negativo) sia di tipo "circolare" (lutto complicato), caratterizzazione che fa assumere a questo processo un andamento compulsivo [22], come rappresentato in Figura 1.

Lo scenario proposto determinerà la fissazione nella memoria emozionale del bambino dell'evento traumatico (perdita), che ritroveremo inalterato nelle sue determinanti affettive in epoca adulta. Possiamo "leggere" questa precoce situazione deprivante come impegno psicodinamico circolare tra gli stadi A e B, senza possibilità da parte dell'Io infantile di un positivo superamento del secondo stadio (avvio dell'elaborazione depressiva del lutto). La rottura del rapporto nelle successive relazioni adulte riattiverà nella personalità stalker l'antica ostilità, odio e aggressività distruttiva verso il partner femminile (madre abbandonica), arcaici vissuti che riattualizzati daranno origine al comportamento compulsivo (aggressività e accanimento).

\section{COAZIONE A RIPETERE}

La coazione a ripetere indica la continua riproduzione nel presente di modelli relazionali connessi a figure affettivamente significative del passato (particolarmente la madre), modalità attraverso la quale un individuo cerca di superare un conflitto infantile irrisolto, organizzando situazioni relazionali similari a quelle che provocarono la precoce lacerazione psichica (p.e. l'ansia abbandonica). Galimberti definisce questo processo come una «tendenza coercitiva e irrazionale che spinge l'indivi- 
duo a mettere in atto determinati comportamenti di cui egli stesso riconosce l'inutilità e l'inadeguatezza, ma la cui mancata esecuzione provoca in lui una sensazione di angoscia» [23].

La coazione a ripetere, quindi, ha una sua tipica funzione riparativa, rintracciata nel tentativo di curare nell'attualità un'antica ferita, dinamica dove il comprendere per superare viene sostituto dall'agire sull'oggetto. Questo tentativo pone l'individuo nella condizione di ricercare inconsciamente una relazione difficoltosa con partner che, "assomigliando" al modello materno frustrante (identificazione), riproduce e fa percepire l'ansia di una rottura relazionale, un costante sentimento di instabilità, la paura dell'allontanamento del partner.

In queste situazioni l'aspetto sessuale della relazione appare secondario, mentre la coazione a ripetere sollecita il soggetto a organizzare rituali di controllo, di possesso narcisistico e distruttivo del partner, tesi a evitare il drammatico e insopportabile vissuto di perdita, illusorio tentativo di trovare una soluzione all'antico conflitto agendo sulla realtà attuale.

Affetto dalla sindrome dell'abbandono e del "tradimento" sperimentata nell'infanzia, lo stalker (ri) vive la rottura relazionale come un trauma eccessivamente significativo e di forte impatto emotivo (alto livello di stress, reazioni aggressive, incapacità nella gestione della rabbia distruttiva, senso di frustrazione e umiliazione, smarrimento, pensieri ossessivi, acting-out), unitamente al fatto che l'assenza-abbandono del partner femminile viene vissuta come una minaccia di annientamento e di annullamento del Sé.

\section{AGGRESSIVITÀ}

L'aggressività si sviluppa dalle relazioni interpersonali, e particolarmente nei rapporti carenti di affettività. Muovendo dai concetti di attaccamento (Bowlby) e dello sviluppo e realizzazione del sé (Kohut), de Zulueta definisce l'aggressività come un comportamento reattivo a rotture traumatiche dell'attaccamento (maltrattamento, rifiuto, abbandono) [24]. Tali fratture psichiche rimuoverebbero nel bambino la sicurezza affettiva, favorendo una reazione di paura espressa attraverso l'aggressività: il trauma (rottura dell'attaccamento) impedisce la possibilità di costruire un sé integro e positivo, trasformando il soggetto da vittima in aggressore. La dinamica argomentata metterà il bambino nella condizione di non poter integrare adeguatamente le fantasie narcisistiche di grandiosità e onnipotenza che, agite nell'angusto spazio della "realtà" sono continuamente frustrate, generano "rabbia narcisistica" che viene espressa attraverso un'aggressività finalizzata alla distruttività [25]. In questa prospettiva l'aggressività è quindi conseguente alla ferita narcisistica, e sarà maggiormente violenta quanto più forte sarà l'investimento sull'organizzazione narcisistica.

Alla base di ogni forma di deprivazione vi è quindi un'aggressività distruttiva dettata dalla paura di non essere amati. Un'ultima e importante annotazione riguarda la posizione di Adler, autore che vede l'aggressività come espressione del bisogno di potenza dell'individuo, comportamento attraverso cui lo stesso tenta di compensare un forte sentimento di inferiorità [26].

\section{NARCISISMO}

Si definiscono narcisistiche le personalità organizzate intorno al mantenimento della propria autostima tramite le conferme provenienti dall'esterno. L'esame delle precoci dinamiche sottostanti a questa struttura evidenzia come fattore centrale una specifica forma di rabbia e di risentimento contro un oggetto (madre) di cui il bambino ha bisogno, ma che vive come frustrante e rifiutante. La cosa desiderata diventa così anche fonte di sofferenza. Per reagire a questa sofferenza il bambino sviluppa un desiderio cosciente o inconscio di distruggere, di rompere e di prendersi con la forza ciò che gli viene rifiutato. I soggetti con problemi narcisistici vivono così la normale reciprocità delle relazioni umane come uno sfruttamento e un'invasione.

Una lettura psicodinamica dei comportamenti di stalking fondata sulla patologia narcisistica è stata proposta da Meloy [27]. Secondo l'autore l'inizio delle molestie avrebbe alla base una fantasia narcisistica di un legame speciale con l'oggetto, illusorio convincimento che deflagra nel contatto con la realtà relazionale adulta (rifiuto o abbandono), suscitando nello stalker sentimenti di profonda vergogna e umiliazione. Insostenibili per tale personalità, questi vissuti causerebbero pesanti reazioni difensive (aggressività, rabbiosità, ostilità, invidia), tipici tratti della patologia narcisistica e borderline.

Alcuni ricercatori hanno suggerito che gli stalker evidenziano un'alta frequenza di perdita dell'oggetto di attaccamento nell'infanzia [28], dati che confermerebbero l'ipotesi della centralità della patologia narcisistica in questo tipo di personalità. L'implicazione dell'assetto narcisistico nel fenomeno stalking, peraltro, ha trovato in letteratura diverse verifiche. Alcune ricerche ritengono che $\mathrm{i}$ quadri psicopatologici più comuni, e i più attendibili fattori predittivi di recidiva nello stalker, includano la presenza di disturbi della personalità appartenenti al "Cluster B", vale a dire il disturbo narcisistico, quello antisociale e quello borderline [29-31].

La struttura narcisistica dello stalker mostra quindi un carattere prevalentemente regressivo sul 
quale si innesta la "coazione a ripetere", intesa come desiderio di riscatto di un'antica sconfitta affettiva patita nell'infanzia. Il comportamento di tale soggetto sarebbe così guidato da gravi disturbi dell'attaccamento con sentimenti che oscillano tra l'idealizzazione e la rabbiosa svalutazione dell'oggetto (l'antica figura rivissuta nel partner attuale), mentre le molestie persistenti rappresenterebbero un tentativo di difesa dalla ferita narcisistica provocata dall'abbandono.

\section{PROCESSO DI SEPARAZIONE- INDIVIDUAZIONE}

La Separazione-Individuazione si svolge dal $4^{\circ}-5^{\circ}$ mese al $36^{\circ}$, configurandosi come un importante processo volto alla crescita psico-relazionale del bambino. Mahler e collaboratori sostengono che: «La fase di separazione-individuazione è caratterizzata da un costante aumento della consapevolezza della separazione fra Sé e "l'altro", che coincide con il sorgere di una sensazione del Sé, di una vera relazione oggettuale e della consapevolezza di una realtà nel mondo esterno» [32].

In questa dinamica il bambino si separa dal rapporto fusionale con la madre (indifferenziazione delle identità), per raggiungere lo stadio di individuazione (identità personale differenziata dalle altre). Il completamento di questo delicato processo facilita e permette l'acquisizione dell'autonomia (fisica, psicologica e cognitiva), primo momento di un funzionamento soggettivo al riparo da pesanti influenze dell'ambiente (madre). Il non completamento di questo processo preclude al bambino (poi ragazzo e adulto) di poter funzionare con i "pezzi personologici" a disposizione, dovendo necessariamente funzionare attraverso "pezzi personologici altri (da sé)", ai quali è inesorabilmente vincolato per non avvertire l'angoscia di frammentazione (dell'lo).

«È soltanto dopo che la costanza oggettuale comincia a stabilizzarsi, [...] che la madre durante la sua assenza fisica può essere sostituita almeno in parte dalla presenza di un'immagine interna sicura che rimanga relativamente stabile indipendentemente dallo stato di bisogno istintuale e di disagio interno. Sulla base di questa conquista la separazione temporanea può essere prolungata $\mathrm{e}$ tollerata meglio» [32].

Il processo di internalizzazione della costanza dell'oggetto (materno) richiede un adeguato superamento del processo di separazione-individuazione, mentre la deficitarietà di tale dinamica prevede angosce infantili conseguenti al pericolo di separazione e di perdita, che scatenano nel bambino terrore del vuoto e intense emozioni di rabbia distruttiva e di umiliazione [33].

Kernberg collega inizialmente la patogenesi della sindrome borderline con lo schema evolutivo di
Mahler, e sottolinea l'importanza dell'aspetto costituzionale (eccesso di aggressività orale), evidenziando le problematiche materne legate alla funzione genitoriale [34]. Questi pazienti, secondo Kernberg, avrebbero superato la fase simbiotica ma non quella della separazione-individuazione. La minaccia all'unitarietà del Sé avvertita dallo stalker nel momento della rottura della relazione (separazione dal partner), testimonia una precaria risoluzione del processo di separazione-individuazione. Da quel momento l'angoscia sollecitata non viene avvertita come una conseguenza della precarietà della propria architettura personologica, bensì quale reattività all'importanza rivestita dal partner, come amore verso questa persona, come indispensabilità di quella relazione. La valutazione del contesto relazionale attraverso antichi criteri fusionali (indifferenziati) rende intricato e irrisolvibile il conflitto, che tende a perpetuarsi con modalità compulsive (accanimento, persecutorietà, ecc).

Non pare quindi accidentale che il narcisismo del borderline - psicopatologia frequentemente correlata allo stalking - sia relativo a un disturbo dello sviluppo nella fase di separazione-individuazione, quindi precedente la strutturazione del SuperIo (norme, divieti, ecc). L'immaturità dello stalker si evidenzia palesemente nella dinamica che segue alla rottura del rapporto, e nei vissuti abbandonici che in questa personalità si fanno largo tra maglie antiche, che hanno ormai assunto profili psicopatologici. L'immaturità di questa struttura può essere agevolmente testimoniata: di fatto solo i bambini possono essere abbandonati, mentre gli adulti vengono lasciati. Nessun adulto, peraltro, viene abbandonato sulle scale di una chiesa o di un convento.

Tale inoppugnabile assunto rende oltremodo comprensibile le caratteristiche dello stalker, la sua profonda immaturità nelle competenze relazionali e nell'assetto affettivo, la sua impulsività, le sue limitate capacità cognitive. In questa prospettiva, la donna scelta dallo stalker sembra avere caratteristiche "materne", che permetterebbero una fantasiosa riparazione psichica dell'antica ferita narcisistica. L'atteggiamento infantile di questa personalità deriverebbe così dal bisogno di essere (figlio) unico, quindi al centro dell'attenzione della loro donna (anticamente madre).

\section{MECCANISMI DIFENSIVI PRINCIPALMENTE UTILIZZATI DALLA PERSONALITÀ STALKER}

I meccanismi di difesa rappresentano le attività (solitamente inconsce) che le funzioni dell'Io utilizzano per bloccare, cambiare, annullare o contenere vissuti che producono tensione, angoscia, dolore, abbandono, ecc. [35]. 
I meccanismi di difesa della personalità stalker paiono volgere un ruolo significativo in questo comportamento, prendendo forma nella negazione, nell'idealizzazione-svalutazione, nella proiezione della colpa sulla vittima [36].

Partendo dall'assunto che nello stalker i confini tra Sé e Altro sono poco definiti (intensità dei bisogni simbiotici, precarietà nel processo di separazione-individuazione), sulla donna attuale (partner) si riverbera (proiezione) l'antico trauma vissuto nella relazione con la figura materna (sentimento di possesso della madre-donna, derivante dal presupposto che la madre è l'unica donna che non può permettersi di lasciare un figlio: lasciare il figlio diviene un "tradimento" che non è possibile elaborare da parte di un bambino). Questo vissuto dà luogo a un'intensa polarizzazione ideo-affettiva su meccanismi di idealizzazione-svalutazione, di onnipotenza e di rabbia nei confronti del partner (meccanismi proiettivi - proiezione dell'angoscia, vale a dire dell'oggetto madre interno negativo identificato nel partner - e anaclitici, vale a dire dipendenza dall'oggetto madre-buono). La proiezione rappresenta quindi la mancanza di un confine psicologico tra il Sé e il mondo esterno (non Sé) per cui qualcosa di interno viene considerato proveniente dall'esterno.

Un altro meccanismo difensivo utilizzato dallo stalker è la negazione. Attraverso questo processo psichico il soggetto, negando la realtà spiacevole che gli ha provocato sofferenza, cerca di sottrarsi a questo spiacevole vissuto. La negazione è una difesa di frequente osservazione nei bambini. Tale condizione deriva da due specifici presupposti decisamente interessanti per il lavoro: governato dal principio del piacere il bambino non è in grado di tollerare e rimandare frustrazioni troppo forti, la sua capacità di un adeguato esame di realtà è inesistente o limitata. Le considerazioni svolte permettono così di affermare che la consistenza della negazione è direttamente proporzionale al grado di immaturità e di fragilità dell'Io.

Un ulteriore meccanismo utilizzato viene rintracciato nella scissione (dell'lo), vale a dire la separazione degli aspetti buoni e degli aspetti cattivi di un "oggetto". Tale dinamica difensiva genera nel soggetto un ambivalente vissuto: la presenza di una parte cattiva all'interno di un oggetto sentito anche buono, inestricabile duplicità avvertita come intollerabile e minacciosa.

Questo processo, che non consentirà al futuro stalker di tollerare l'emergere di sensazioni negative o il contatto con "oggetti" interni cattivi (vuoto, rabbia, angoscia, inquietudine), si formerebbe per un eccesso di frustrazione dei bisogni pulsionali primari del bambino, in una fase dello sviluppo in cui lo stesso non è in grado di tollerarla [37]. In questa situazione, rifiutata e primitiva, l'aggressività infantile non può assumere il profilo di un'energia matura utilizzabile in epoca adulta, aumentando la probabilità di agiti aggressivi anche gravi (discontrollo degli impulsi).

Inoltre, muovendo dal presupposto che durante $\mathrm{i}$ primi tre anni nel bambino si organizzano rappresentazioni reali e quindi condivisibili, le difficoltà dello stalker potrebbero derivare dal fatto che il suo sistema rappresentazionale è costituito da oggetti sdoppiati con significati variabili, quindi inutilizzabili per costruire vissuti efficaci, reali e saldi. I meccanismi difensivi argomentati, stante la loro arcaica temporalità (periodo pre-edipico), suggeriscono una speculare arcaicità in alcuni aspetti psico-comportamentali dello stalker, personalità bloccata in una remota circolarità psicodinamica.

\section{- LA PERSONALITÀ STALKER VALUTATA ATTRAVERSO IL MMPI}

I casi osservati dall'autore sono stati analizzati attraverso il MMPI (Minnesota Multiphasic Personality Inventory) [38], questionario che fornisce una valutazione oggettiva delle caratteristiche di personalità del soggetto, dei suoi sintomi e dei suoi atteggiamenti personali, la cui rilevanza dal punto di vista del profilo clinico e della prognosi è stata dimostrata da numerosi studi di ricerca [39]. Le risultanze testologiche del profilo (media delle scale dei MMPI) elaborate con il sistema "Due-Plus" [40] sono presentate di seguito.

\section{INTERPRETAZIONI PER SCALE E INDICI SPECIALI DEL MMPI}

Il soggetto tende a reagire depressivamente di fronte a eventi anche solo marginalmente frustranti, e a generalizzare tale vissuto anche ad altre esperienze. Questa visione negativa di sé, del mondo, del futuro, può accompagnarsi a modalità di pensiero dicotomico, scarsamente flessibile, riducendo ulteriormente le sue capacità di affrontare efficacemente situazioni problematiche. Tale quadro può facilmente tradursi in atteggiamenti di "rinuncia" e sensi di inutilità. Sono presenti marcate note di critica, oppositività e ostilità, che possono anche sfociare in atteggiamenti manifestamente aggressivi. Il soggetto appare molto intollerante alla frustrazione cui tende a reagire spesso impulsivamente e inadeguatamente. Può essere presente un atteggiamento di costante attribuzione all'esterno delle cause delle proprie frustrazioni, nelle quali peraltro il soggetto incorre facilmente a causa di una notevole immaturità di giudizio. I rapporti interpersonali necessariamente estensivi e poco duraturi, risentono negativamente dell'oppositività da cui sono caratterizzati. 


\section{INTERPRETAZIONI DELLE VARIABILI DI DIAMOND}

Abulia, sensi di sfiducia, marcata astenia possono influenzare negativamente i livelli di attività del soggetto che, pertanto, può avere notevoli difficoltà a iniziare attività anche di routine. Il soggetto mostra una discreta valutazione di sé e delle proprie capacità; se da un lato ciò lo mette in grado di non evitare le situazioni problematiche, dall'altro può inficiare l'adeguatezza delle soluzioni per la possibile presenza di una certa superficialità nel valutare le circostanze. Tratti di compiacenza formale e di accentuazione di generica disponibilità compaiono nel soggetto come modalità di adattamento prevalentemente autoplastico. Il maggior peso attribuito all'accettazione da parte del gruppo può agire da rinforzo in tal senso. Il soggetto inoltre appare molto reattivo alle frustrazioni, che sembra tollerare solo in misura ridotta.

\section{CODE TYPE (TIPI DI CODICE) DEGLI STALKER ANALIZZATI}

I Code Type del MMPI sono stati elaborati partendo dalla constatazione che determinate elevazioni di scale (maggiori di 70 punti T) si trovano più frequentemente in certe popolazioni che non in altre. Il sistema di interpretazione per codici rappresenta un metodo pratico, capace di riassumere gli elementi più importanti di un profilo, e di individuare l'eventuale quadro morboso con un numero di codice (Code-Type) $[41,42]$. Di seguito vengono presentati i tipi di codice dei soggetti analizzati, unitamente ai tratti psico-comportamentali e psicopatologici a essi correlati.

\section{Soggetto 1: Code Type 4-2 (Pd-D)}

Il soggetto mostra marcati sentimenti depressivi, difficoltà nel differire il soddisfacimento degli impulsi, intolleranza per gli standard e i valori sociali. Spesso è pervaso da tensione, incapacità a tollerare la noia e dalla convinzione che gli altri siano ostili nei suoi confronti. Nel rapporto interpersonale si mostra ostile e irritabile, tende a manipolare gli altri e, dietro una facciata di competenza e sicurezza, cela sentimenti di insoddisfazione e difficoltà. Evidenzia una marcata incapacità nel mantenere relazioni durature, strette e responsabili con familiari, partner e amici e ciò determina relazioni improntate a una caratteristica oscillazione tra aggressività e passività. Può reagire alle situazioni di stress rifugiandosi nell'alcolismo o nell'assunzione di droghe e, in seguito, mostrare sentimenti di colpa e rimorso. Può ri- chiedere aiuto specialistico, ma questa richiesta non sembra sostenuta da elementi attendibili: la prognosi per un approccio psicoterapico non è positiva. Le persone con questo codice sono classificate tipicamente come passivo-dipendenti e ricevono la diagnosi di disturbo antisociale di personalità.

\section{Soggetto 2: Code Type 4-2-5 (Pd-D-Mf)}

Il soggetto mostra marcati sentimenti depressivi, difficoltà nel differire il soddisfacimento degli impulsi e intolleranza per gli standard e i valori sociali. Nel rapporto interpersonale si mostra ostile e irritabile, tende a manipolare gli altri e, dietro una facciata di competenza e sicurezza, cela sentimenti di insoddisfazione e difficoltà. Appare incapace di mantenere relazioni durature, strette e responsabili con familiari, partner e amici e ciò determina relazioni improntate a una caratteristica oscillazione tra aggressività e passività. Può reagire alle situazioni di stress rifugiandosi nell'alcolismo o nell'assunzione di droghe e, in seguito, mostrare sentimenti di colpa e rimorso. Le persone con questo codice manifestano atteggiamenti improntati a immaturità e narcisismo. Sono possibili irregolarità della condotta sessuale con ricerca di esperienze devianti: la propria struttura psicosessuale appare scarsamente aderente al modello di sessualità convenzionale. Può richiedere aiuto specialistico ma questa richiesta non sembra sostenuta da elementi attendibili: la prognosi per un approccio psicoterapico non è positiva. Le persone con questo codice sono classificate tipicamente come passivo-dipendenti e ricevono la diagnosi di disturbo antisociale di personalità.

\section{Soggetto 3: Code Type 4-2-7 (Pd-D-Pt)}

è presente una condizione di rabbia intensa sottostante a sentimenti di inferiorità, fallimento, immobilità e ambivalenza. Sono presenti evidenti sintomi depressivi con ansia e spunti fobici. Il soggetto tende a mostrarsi arrabbiato, ostile, immaturo, orale-dipendente con forti bisogni di attenzione e sostegno e può manifestare problemi relativi all'autocontrollo: sono possibili infatti episodi di acting-out, un orientamento verso comportamenti socialmente disapprovati con capacità di controllo difettuali e mancanza di insight. È un tipo di codice piuttosto frequente tra i soggetti che fanno abuso di alcool e/o altre sostanze e, soprattutto, che vivono difficoltà familiari. La diagnosi più comune assegnata ai soggetti con questo tipo di codice è quella di reazione depressiva su una base di personalità orientata in senso passivo-aggressivo o antisociale. 


\section{CONCLUSIONI}

Dall'osservazione e dalle risultanze psicometriche (MMPI) ottenute dagli stalker osservati è possibile trarre alcune importanti considerazioni che, pur non generalizzabili (esiguità del campione), paiono comunque un attendibile orientamento sull'operatività diagnostica, farmacologica e psicoterapeutica più funzionali in questo fenomeno.

La mappa mentale dello stalker (e quindi anche quella comportamentale) pare assemblata su prototipici e negativi costrutti relazionali sperimentati con la figura materna (vale a dire una figura femminile). Di fatto, la presenza nell'infanzia di una "madre rifiutante" produce un attaccamento insicuro, imprinting relazionale che si riverbererà pesantemente sulle successive esperienze di natura affettiva (coazione a ripetere). Nella personalità in esame, il rifiuto insito nella comunicazione di rottura del rapporto formulato dal partner, riattiva le antiche tracce attualizzando questo arcaico lacerante vissuto, situazione che fa scattare il comportamento persecutorio (rabbia agita).

Riferendosi a un profilo psicodinamico appartenente alla madre, lo stalker - deficitario nel processo di separazione-individuazione (precarietà nei confini identitari) - non è in grado di porre una demarcazione psichica capace di differenziare "madre" da "partner", artificiosa e patologica coincidenza che permette alle antiche e rabbiose richieste infantili di convergere immutate nell'attualità relazionale. Per tale ragione il vissuto dello stalker oscilla da momenti di idealizzazione del partner (vissuto come madre buona) a momenti di aggressività distruttiva verso questa dimensione (vissuta come madre abbandonica).

Nelle dinamiche esposte nel lavoro possiamo riscontrare in questa personalità una bassa soglia di tolleranza alla frustrazione e, conseguentemente, un'entrata violenta degli impulsi nella relazione, la precarietà o l'assenza di controllo verso $\mathrm{i}$ sentimenti provocati dalla frustrazione (rifiuto del partner di continuare la relazione), che producono irritabilità, impulsività, aggressività, angoscia, panico, distruttività. In particolare, le variabili che meglio differenziano lo stalker fisicamente violento rispetto a quello non violento sono la rabbia, la vendetta, l'eccitazione emotiva, l'umiliazione, la proiezione della colpa, e la patologia derivante da un attaccamento insicuro [43].

La personalità stalker evidenzia di non essere in grado di bloccare la pressante urgenza degli impulsi, incapacità che determina una profonda disorganizzazione dell'lo, che a sua volta produce l'impossibilità di arginare il fulmineo e disordinato aumento dell'intrusività delle emozioni, la cui distorta interpretazione appesantisce notevolmente il significato a esse soggettivamente assegnato. Inoltre, la non consapevolezza di un comportamento che segue le leggi della "coazione a ripetere", pone lo stalker nella condizione di vivere come attuale un evento perso nella notte della deprivazione infantile, unitamente alla profonda difficoltà di leggere con precisione le coordinate che legano la causa agli effetti all'interno della relazione interpersonale.

Queste organizzazioni personologiche, dato il forte bisogno di appoggio, evidenziano modalità relazionali dove prevale la necessità di non perdere il contatto con l'oggetto buono e rassicurante, strategia tesa a evitare le fortissime angosce di abbandono, di perdita di significato, di svuotamento, di frammentazione. Per queste ragioni, il modo di interagire e di stabilire relazioni da parte dello stalker rispecchia una condizione di dipendenza passiva e insieme di manipolazione del partner, che questa personalità tenta di non perdere, di controllare e di dominare.

\section{LA LEGISLAZIONE ITALIANA}

Lo stalking è regolato dal Decreto Legge n. 11 del 23 febbraio 2009 "Misure urgenti in materia di sicurezza pubblica e di contrasto alla violenza sessuale, nonché in tema di atti persecutori", che stabilisce che:

- il reato è di tipo penale;

- il reato è perseguibile a querela della vittima. Si procede d'ufficio nel caso il fatto colpisca un minore o una persona disabile, quando il fatto comporti un altro delitto per il quale si proceda d'ufficio oppure se l'autore delle molestie è già stato ammonito dal questore;

- per sostenere le vittime è istituito un numero verde nazionale, che fornisce assistenza psicologica e giuridica, e permette di segnalare (su richiesta della vittima) le molestie alle forze dell'ordine. Sono inoltre presenti sul territorio dei centri dei centri anti-stalking;

- durante il processo penale contro il molestatore, a questi può essere vietato di avvicinarsi alla vittima e ai luoghi che questa frequenta;

- la pena per chi viene ritenuto colpevole di atto persecutorio è una condanna che va dai sei mesi ai quattro anni di reclusione. La pena può essere più aspra se il fatto è stato commesso da un coniuge, legalmente separato o divorziato, da persona che sia stata legata da relazione affettiva, o da persona già ammonita dal questore. Inoltre la pena viene inasprita se il fatto è commesso contro un minore, una donna in gravidanza o un disabile. 
L'organizzazione personologica che presiede e sollecita lo stalking si mostra quindi impotente di fronte al rifiuto, all'abbandono, alla paura, al disagio, all'angoscia, evidenziando di disorganizzarsi anche per frustrazioni modeste, sentimenti verso i quali lo stalker mette in atto meccanismi regressivi che si manifestano attraverso lo sviluppo di fantasie di controllo (potenza, forza e invulnerabilità), nel sottoporre il partner a limitazioni forzate del suo spazio vitale, nell'attuare una serie di comportamenti di accanimento volti a produrre nell'altro una "vita in angosciosa apnea esistenziale".

\section{DISCLOSURE}

L'Autore dichiara di non avere conflitti di interesse di natura finanziaria.

\section{BIBLIOGRAFIA}

1. Pathé M, Mullen P. The impact of stalkers on their victim. British Journal of Psychiatry 1997; 170: 12-7

2. Krammer A, Stepan A, Baranyi A, Kapfhammer HP, Rothenhäusler HB. The effects of stalking on psychiatrists, psychotherapists and psychologists. Prevalence of stalking and its emotional impact. Nervenarzt 2007; 78: 809-17

3. Meloy JR, Gothard S. Demographic and clinical comparison of obsessional followers and offenders with mental disorders. Am J Psychiatry 1995; 152: 258-63

4. Meloy JR. The psychology of stalking. Accademic Press: San Diego (California), 1998

5. Tjaden P, Thoennes N. Stalking in America: findings from the National Violence Against Women Survey: research in brief. Washington, DC: US Department of Justice, National Institute of Justice Centers for Disease Control and Prevention, April 1998

6. Galeazzi GM, Curci P. Sindrome del molestatore assillante (stalking): una rassegna. Giornale Italiano di Psicopatologia, 2001; 7: 434-52

7. Gargiullo BC, Damiani R. Lo stalker, ovvero il persecutore in agguato. Classificazione, assessment e profili psicocomportamentali. Milano: Franco Angeli, 2008

8. Lattanzi, M, Ferrara G, Mascia I, Oddi G. Stalking: La ricerca italiana e internazionale. In: Lattanti M, Ferraris OM (a cura di). Stalking. Il lato oscuro delle relazioni interpersonali. Roma: Ediservice, 2003; pp. 64-71

9. Osservatorio Nazionale Stalking, www.stalking.it

10. Ashmore R, Jones J, Jackson A, Smoyak S. A Survey of Mental Health Nurses' Experiences of Stalking.J Psychiatr Ment Health Nurs 2006; 13: 562-9

11. Morrison KA. Predicting violent behavior in stalkers - A preliminary investigation of Canadian cases in criminal harassment. $J$ Forensic Sci 2001; 46: 1403-10

12. Bowlby J. La separazione dalla madre (Vol. 2). Torino: Bollati Boringhieri, 1975

13. Kobak RR, Sceery A. Attachment in late adolescence: working models, affect regulation, and representation of se and others. Child Dev 1988; 59: 135-46

14. Wilson J, Ermshar A, Weish R. Stalking as paranoid attachment: a typological and dynamic model. Attach Hum Dev 2006; 8: 13957

15. MacKenzie RD, Mullen PE, Ogloff JR, McEwan TE, James DV. Parental bonding and adult attachment styles in different types of stalker. Forensic Sci 2008; 53: 1443-9

16. Lewis SF, Fremouw WJ, Del Ben K, Farr C. An investigation of the psychological characteristics of stalkers: empathy, problemsolving, attachment and borderline personality features.J Forensic Sci 2001; 46: 80-4

17. Lyons-Ruth K, Repacholi B, McLeod S, Silva E. Disorganized attachment behaviour in infancy: Short-team stability, maternal and infant correlates and risk-related subtypes. Dev Psychopathol 1991; 3: 377-96

18. Allen JP, Hauser ST, Borman-Spurrell E. Attachment theory as a framework for understanding sequelae of severe adolescent psychopathology: an 11-year follow-up study. J Consult Clin Psychol 1996; 64: 254-63

19. Holtzworth-Munroe A, Hutchinson G, Stuart GL. Attachment patterns of martially violent versus nonviolent men: Data from the adult attachment interview. Paper presented at the Association for the Advancement of Behavior Therapy, Boston, 1992

20. Fonagy P, Leigh T, Steele M, Stele H, Kennedy R, Mattoon G et al. The relation of attachment status psychiatric classification, and response to psychotherapy. J Consult Clin Psychol 1996; 64: 22-31

21. Zetzel E, Meissner WW. Psichiatria psicoanalitica. Torino: Bollati Boringhieri, 1978

22. Infrasca R. Il disturbo distimico. Una rivisitazione del processo di lutto. Quaderni italiani di Psichiatria 1990; 9: 469-80

23. Galimberti U. Dizionario di Psicologia. Torino: Utet, 1994

24. de Zulueta F. Dal dolore alla violenza: le origini traumatiche dell'aggressività. Milano: Cortina, 1999

25. Kohut H. Pensieri sul narcisismo e sulla rabbia narcisistica. In: Kohut H. La ricerca del Sé. Torino: Bollati Boringhieri, 1982 
26. Adler A. Il temperamento nervoso. Roma: Astrolabio, 1950

27. Meloy JR. Stalking: an old behavior, a new crime. Psych Clin North Am 1999; 22: 85-99

28. Kienlen KK, Birmingham DL, Solberg KB, O’Regan JT, Meloy JR. A comparative study of psychotic and nonpsychotic stalking. J Am Acad Psychiatry Law 1997; 25: 317-34

29. Meloy J. Stalking and violence. In J. Boon and L. Sheridan (a cura di). Stalking and psychosexual obsession: Psychological perspectives for prevention, policing, and treatment. West Sussex (UK): John Wiley \& Sons, Ltd, 2002

30. Rosenfeld B. Recidivism in stalking and obsessional harassment. Law Hum Behav 2003; 27: 251-65

31. Schneider C, Tress W. Stalking: unwelcome company. The psychopathology of a modern phenomenon. MMW Fortschr Med 2005; 147: 30-3

32. Mahler MS, Pine F, Bergman A. La nascita psicologica del bambino. Torino: Bollati Boringhieri, 1978

33. Gaddini R. Patologia psicosomatica come difetto maturativo. Rivista di Psicoanalisi 1980; 26: 381-7

34. Kernberg O. Borderline conditions and pathological narcissism. New York: Jason Aronson, 1975

35. Lingiardi V, Madeddu F. I meccanismi di difesa. Teoria clinica e ricerca empirica. Milano: Cortina, 1994

36. Skoler G. The archetypes and psychodynamics of stalking. In: JR Meloy (a cura di). The psychology of stalking: clinical and forensic perspectives. New York: Academic Press, 1998; pp. 85-112

37. Kernberg O. Sindromi marginali e narcisismo patologico. Torino: Bollati Boringhieri, 1978

38. Hathaway SR, Mckinley JC. The Minnesota Multiphasic Personality Inventory Manual (revised). New York: Psychological Corporation, 1951

39. Dahlstrom W, Welsh GS, Dahlstrom LE. An MMPI handbook (2 vol.) Minneapolis: University of Minnesota press, 1982

40. Mosticoni R, Mosticoni S. Sistema Due-Plus. Roma: M-TWO, 2005

41. Marks PA, Seeman W, Haller DL. The actuarial use of the MMPI with adolescents and adults. Baltimore: Williams \& Wilkins, 1974

42. Archer RP, Pancoast DL, Klinefelter D. A comparison of MMPI code types produced by traditional and recent adolescent norms. J Consult Clin Psychol 1989; 1: 23-9

43. Morrison KA. Differentiating between physically violent and nonviolent stalkers: an examination of Canadian cases. J Forensic Sci 2008; 53: 742-51

\section{CORRESPONDING AUTHOR}

Dott. Roberto Infrasca; e-mail: roberto.infrasca@asl5.liguria.it 